\title{
Thin-Sheet Inversion Modeling of Geomagnetic Deep Sounding Data Using MCMC Algorithm
}

\author{
Hendra Grandis, ${ }^{1}$ Michel Menvielle, ${ }^{2}$ and Michel Roussignol ${ }^{3}$ \\ ${ }^{1}$ Faculty of Mining and Petroleum Engineering, Bandung Institute of Technology, Jalan Ganesha 10, Bandung 40132, Indonesia \\ ${ }^{2}$ Centre d'Etude de l'Environnement Terrestre et Planétaires, 3 avenue de Neptune, 94107 Saint-Maur-des-Fosses, France \\ ${ }^{3}$ Laboratoire d'Analyse et de Mathématique Appliquée, Université de Marne la Vallée, 5 boulevard Descartes, \\ 77454 Marne-la-Vallée, France
}

Correspondence should be addressed to Hendra Grandis; grandis@earthling.net

Received 31 May 2013; Accepted 18 September 2013

Academic Editor: Vincenzo Lapenna

Copyright (c) 2013 Hendra Grandis et al. This is an open access article distributed under the Creative Commons Attribution License, which permits unrestricted use, distribution, and reproduction in any medium, provided the original work is properly cited.

The geomagnetic deep sounding (GDS) method is one of electromagnetic (EM) methods in geophysics that allows the estimation of the subsurface electrical conductivity distribution. This paper presents the inversion modeling of GDS data employing Markov Chain Monte Carlo (MCMC) algorithm to evaluate the marginal posterior probability of the model parameters. We used thin-sheet model to represent quasi-3D conductivity variations in the heterogeneous subsurface. The algorithm was applied to invert field GDS data from the zone covering an area that spans from eastern margin of the Bohemian Massif to the West Carpathians in Europe. Conductivity anomalies obtained from this study confirm the well-known large-scale tectonic setting of the area.

\section{Introduction}

In geomagnetic deep sounding (GDS), we measure the natural Earth's magnetic transient variations to infer largescale subsurface conductivity distribution. Recent advances in magnetotelluric (MT) technique tend to put the attention to move towards local scale investigation of conductivity anomalies with more economic interests as in exploration for mineral, geothermal, or hydrocarbon. However, GDS is still considered as the most appropriate natural source electromagnetic (EM) method capable of imaging the Earth's interior especially for tectonic study at the regional and continental scales (e.g., $[1,2])$.

This paper describes the inversion modeling technique for GDS data in terms of conductivity distribution by using the Markov Chain Monte Carlo (MCMC) algorithm. The MCMC inversion algorithm has been applied for 1D modeling of MT [3, 4], vertical electrical sounding (VES) [5] and also controlled-source audio-frequency MT (CSAMT) [6] with satisfactory results. In the so-called thin-sheet modeling, the conductivity variations are assumed to be confined in a thin layer such that the model parameters are integrated conductivity over the thickness of the thin layer. This simplification suits well for modeling GDS data that are mostly sensitive to lateral variation of conductivity $[7,8]$.

We will first briefly review the concept and the formulation of the thin-sheet modeling and then describe the MCMC inversion algorithm. The result of inversion of real GDS data from Bohemian Massif-West Carpathians and also its interpretation will be discussed.

\section{Thin-Sheet Electromagnetic Modeling}

In electromagnetic (EM) geophysics, the thin-sheet modeling refers to an approximation of 3D conductivity variation by a thin layer with variable conductance, that is, integrated conductivity over the thickness of the thin layer. Such approximation is generally valid in the large-scale studies where the heterogeneities are confined in a layer with thickness much smaller than the penetration depth of EM fields. The thinsheet modeling significantly simplifies the resolution of the Maxwell's equations describing the EM fields in quasi-3D media. We used an algorithm employing integral equation method for thin-sheet EM modeling proposed by Vasseur 
and Weidelt [1]. By using the integral equation method, the computation domain covers only the anomalous zone where the conductance differs from that of normal (homogeneous or stratified) host medium (Figure 1).

The thin-sheet containing heterogeneities is discretized into rectangular uniform blocks in which the conductance is assumed constant. The total electric field in the $k$ th cell located at the heterogeneous layer is expressed by

$$
\mathbf{E}(k)=\mathbf{E}^{n}(k)-i \omega \mu_{0} \sum_{k^{\prime} \in K} \tau_{k^{\prime}} \mathbf{G}\left(k, k^{\prime}\right) \cdot \mathbf{E}\left(k^{\prime}\right),
$$

where $\mathbf{E}^{n}(k)$ is the normal electric field associated with the normal conductivity distribution, $\mathbf{G}\left(k, k^{\prime}\right)$ is the Green kernel representing the electric field at $k$ due to a unitary dipole at $k^{\prime}$, and $\tau_{k}$ denotes the anomalous conductance of the $k$ th block, $k, k^{\prime} \in K$. The $\left(i \omega \mu_{0}\right)$ factors are imaginary part of complex numbers, angular frequency, and magnetic permeability of the free space, respectively. Equation (1) is in fact a system of $M=2 K$ linear equations of complex values of the orthogonal electric fields $\left(E_{x}, E_{y}\right)$ at each block associated with a certain conductance distribution, with $K$ being the total number of blocks [1].

For a large number of blocks in the anomalous domain, a direct matrix inversion to resolve (1) may be unstable and time consuming. Considering its form, (1) is more suitable to be resolved by the Gauss-Seidel method, where an initial value is iteratively updated to obtain the solution. At $(j+1)$ th iteration, the estimated electric field is calculated by

$$
\begin{aligned}
\widehat{\mathbf{E}}(k)_{j+1}= & \frac{1}{1+i \omega \mu_{0} \tau_{k} \underline{\mathbf{G}}(k, k)} \\
& \times\left(\mathbf{E}^{n}(k)-i \omega \mu_{0}\left[\sum_{k^{\prime}=1}^{k-1} \tau_{k^{\prime}} \underline{\mathbf{G}}\left(k, k^{\prime}\right) \cdot \mathbf{E}\left(k^{\prime}\right)_{j+1}\right.\right. \\
& \left.\left.+\sum_{k^{\prime}=k+1}^{K} \tau_{k^{\prime}} \underline{\mathbf{G}}\left(k, k^{\prime}\right) \cdot \mathbf{E}\left(k^{\prime}\right)_{j}\right]\right) .
\end{aligned}
$$

The normal electric field $\mathbf{E}^{n}(k)$ is commonly used as the initial value for $\mathbf{E}(k)$, that is, $\mathbf{E}(k)_{0}$. A relatively fast convergence can be reached since at the subsequent iterations all updated elements of the solution $\left(\mathbf{E}\left(k^{\prime}\right)_{j+1}\right.$ for $\left.k^{\prime} \neq k\right)$ are used to estimate the remaining elements. For low to moderate conductivity contrast, convergent solution is generally achieved after 10 to 20 iterations [9].

Resolving (1) results in electric fields $\left(E_{x}, E_{y}\right)$ that can then be used to calculate the corresponding orthogonal magnetic fields $\left(H_{x}, H_{y}, H_{z}\right)$. In the MT method the transfer function relating electric and magnetic fields is the complex impedance tensor, while in the GDS technique we are interested in the relationship between vertical and horizontal magnetic fields. The complex magnetic transfer function is usually presented as magnetic (real and imaginary) induction vectors using the following equations:

$$
H_{z}=T_{z x} H_{x}+T_{z y} H_{y}
$$

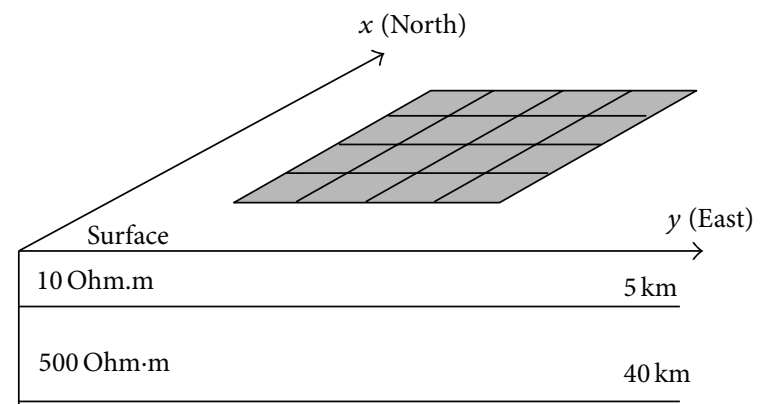

$1000 \mathrm{Ohm} \cdot \mathrm{m}$

\begin{tabular}{ll} 
& $70 \mathrm{~km}$ \\
\hline 10 Ohm.m & \\
$z$ &
\end{tabular}

(arbitrary scale)

FIGURE 1: A heterogeneous superficial thin-sheet model embedded in $1 \mathrm{D}$ or layered host medium. The resistivities and thicknesses are arbitrary, for the illustration purpose only.

$$
\begin{gathered}
\bar{V}_{\mathrm{Re}}=-\operatorname{Re}\left(T_{z x}\right) \hat{x}-\operatorname{Re}\left(T_{z y}\right) \hat{y}, \\
\bar{V}_{\operatorname{Im}}=\operatorname{Im}\left(T_{z y}\right) \hat{x}+\operatorname{Im}\left(T_{z y}\right) \hat{y},
\end{gathered}
$$

where $\hat{x}$ and $\hat{y}$ are unitary vector in the $x$ - and $y$-axes, respectively. Note that in (4a) the direction of the real induction vector is inverted such that it conforms to the Parkinson's induction arrows. With this convention, the real induction arrows point towards the conductive medium [10].

\section{Markov Chain Monte Carlo (MCMC) Inversion}

Assuming that the parameters for 1D host medium are known, the inverse problem consists in determining the conductance of the thin-sheet discretized in blocks (see Figure 1). Furthermore, possible conductances for each thinsheet block are also available as prior information. These conductances are discrete values in a sufficiently large interval covering low to high conductance to minimize bias in the inverse problem resolution. Starting from a homogeneous thin-sheet, the conductance of the $k$ th block is updated by choosing from $N$ "a priori" values $\tau=\left[\tau_{i}\right], i=1,2, \ldots, N$, weighted by the probability

$$
P\left(\tau_{i}\right)=\exp \left(-E\left(\mathbf{m} \mid m_{k}=\tau_{i}\right)\right),
$$

where $E\left(\mathbf{m} \mid m_{k}=\tau_{i}\right)$ is the misfit related to a model $\mathbf{m}$ in which $m_{k}=\tau_{i}$ while conductances of blocks other than $k$ th block are fixed at their current values. Assuming gaussian errors for observed data, more explicit expression for (5) is as follows:

$$
P\left(\tau_{i}\right)=C \exp \left(\sum_{j=1}^{\mathrm{ND}}-\frac{1}{2}\left(\frac{d_{j}-f_{i}\left(\mathbf{m} \mid m_{k}=\tau_{i}\right)}{\sigma_{j}}\right)\right),
$$


where $d_{j}$ and $\sigma_{j}$ are the observed data and their standard deviations, while $f_{j}$ is calculated or theoretical response of the model $\mathbf{m}$ and $j=1,2, \ldots, \mathrm{ND}$ are data indices with ND being the number of data. In the case of GDS, data are the real and imaginary parts of the magnetic vector at each station for frequencies or periods used. $C$ is a normalizing constant for probabilities $P\left(\tau_{i}\right)$ that involves a sum over $N$ possible conductance values.

Equation (6) represents the relative probability for $\boldsymbol{\tau}=$ $\left[\tau_{i}\right], i=1,2, \ldots, N$, as possible values for $m_{k}$. We need to resolve the forward modeling $N$ times before a decision is made to select the best conductance value for each block, which is amenable for a reasonable number of $N$, that is, 10 to 20 . It is obvious that $\tau_{i}$ corresponding to smaller misfit will have greater probability to be selected. However, conductances with greater misfit will still have a chance to be selected; they are only less probable. This characteristic gives the MCMC inversion algorithm an ability to escape local minima frequently encountered in resolving a highly nonlinear inverse problem using linearized approach.

Updating model parameters sequentially using (6) generates a series of models (or states) obeying the fundamental Markov chain rule; that is, probability of a state depends on the past states only through the previous (or immediate past) state. Therefore, the probability in (6) serves as the transition probability of the Markov chain that governs the evolution in "time" or in iterations of the chain in a set of finite possible models. We assume without further details that key characteristics of the chain hold, for example, homogeneity or invariance and ergodicity that assure its convergence to meaningful results. After sufficiently long iterations, the Markov chain is independent of its initial state and exhibits a stationary state described by its invariant probability. It can be shown that the transition probability will be asymptotic to the invariant probability of the constructed Markov chain [11].

The iterative process described earlier is commonly called Gibbs sampler that may be employed to approximate a probability density function (PDF). It leads to the approximation of the marginal posterior probability for model parameter $m_{k}$ given the observed data, which is the solution of the inverse problems recast in the Bayesian inference context [11]. In general, empirical averages tend towards statistical averages such that statistical measures for model parameters (mean and variance) can be estimated from the evaluation of the estimated posterior quantities $[12,13]$.

The MCMC inversion algorithm was tested to invert synthetic data associated with thin-sheet models with satisfactory results [9]. In that test both resistive and conductive anomalies were relatively equally well resolved due to the addition of the Gauss-Seidel iterations in the misfit evaluation. The paper also underlined the importance of properly discretize the "a priori" conductance interval.

\section{Inversion of GDS Field Data}

The geology of the study area covering Czech, Slovakia, and parts of Poland is relatively complex since it is composed of two different geological units, that is, the Bohemian Massif and the western part of the East Carpathians. The Bohemian Massif and the Carpathians are part of the Hercynian and the Alpine chains, respectively, that are fundamental elements of the geology of Europe. Figure 2 shows a simplified tectonic map of the study area, extended to the East to cover the whole Carpathians and to the South to cover the Pannonian Basin. Much of the details about Bohemian Massif in the western part of the study area is not shown on such regional scale and simplified map. The most remarkable geological feature often related to the presence of the West Carpathian conductivity anomaly is the Pieniny Klippen belt. It is a narrow banded zone of extreme shortening and subvertical strike-slip fault zone of hundreds of kilometers long resulting from the convergence of inner Carpathians to the north area [14].

The study of the Carpathian conductivity anomaly has a very long history since its discovery deduced from geomagnetic soundings in the late 70s. Newly acquired data in recent studies still exhibit roughly similar regularities, in particular those related to the West Carpathian anomaly (WCA). Kováčiková et al. [15] employed thin-sheet inversion modeling based on the conjugate gradient minimization of misfit and a stabilizing functional. In order to describe better the sharp tectonic boundaries in this region, a minimum gradient functional was applied. This additional minimization focused the resulting image from their inversion. This approach produced blocky conductivity distribution in relatively narrow anomalous zones. Those conductive zones coincide with well-known conductivity anomalies present in the study area, mainly the Bohemian Massif anomaly (BMA) and the West Carpathian anomaly (WCA).

The GDS data set used in our study is fundamentally the same as previous studies (e.g., [15]), provided by the Institute of Geophysics, Academy of Sciences of the Czech Republic in cooperation with the Université Paris Sud, France (Menvielle, pers. comm.). The observation stations are distributed along several north to south and also northwest to south-east profiles crossing tectonic features of the area characterized by conductivity anomalies, in particular the WCA. Figure 3 shows the outline of the study area and the stations distribution along with country border and WCA outlines. The WGS84 geographical coordinate system used in Figure 3 and in all subsequent maps is for reference position only. For the thin-sheet modeling the area was then discretized into blocks with 35 by $35 \mathrm{~km}$ in size (see Figure 3).

The observed data are magnetic transfer function at 143 single stations for the periods of 20,32,64, and 98 minutes. The data processing is described in [16, 17]. Figure 4 shows the GDS data presented as real and imaginary part of the magnetic induction vectors at the period of 20 minutes. As shown in (4a) the real part vectors were inverted in accordance to Parkinson's convention such that the vectors point towards more conductive zones. From Figure 4, qualitatively we can correlate the crossings of the real magnetic induction vectors with the prominent conductive anomalies present in the study area, especially the West Carpathian anomaly. The GDS data for other periods used in this study, that is, 32,64 , 


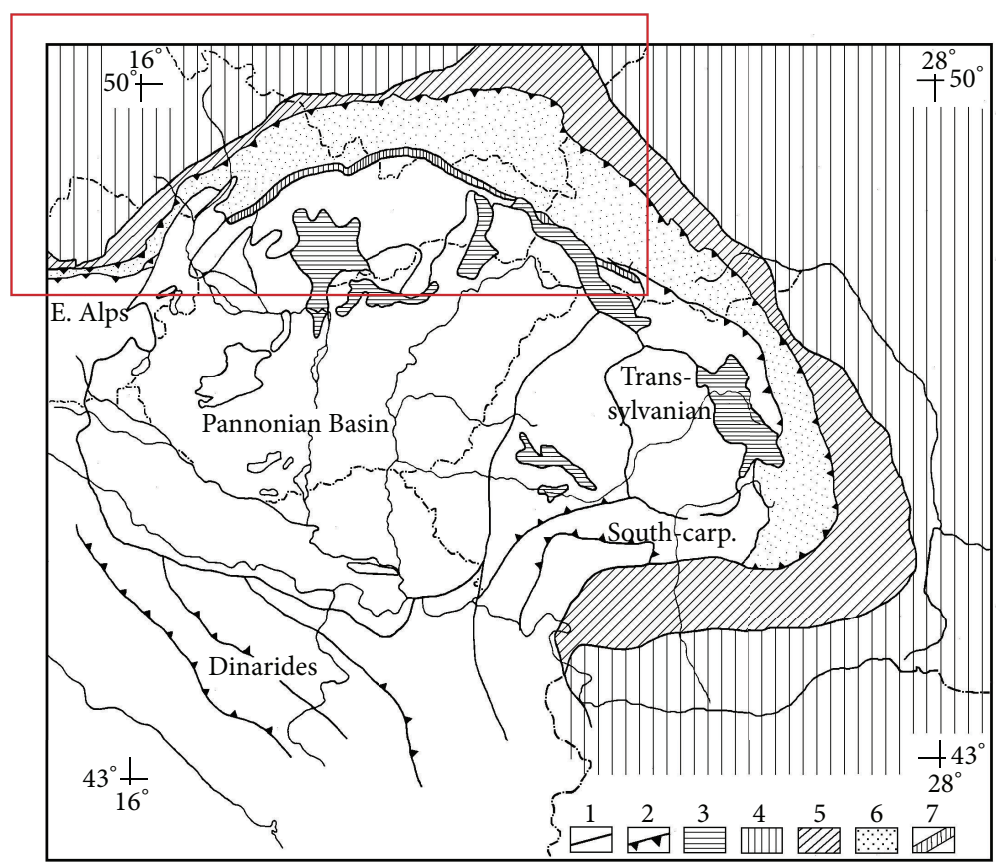

FIGURE 2: Simplified tectonic map of Carpathians and Pannonian Basin (adapted from [14]) in WGS84 geographical coordinates system as reference: (1) limit of structural units, (2) thrusting fronts, (3) neovolcanic rocks, (4) European plate, (5) mollasic foredeep, (6) outer Carpath (Flysch), and (7) Pieniny Klippen belt. Approximate position of the area of this study is outlined by the red box located at the north-west part of the map.

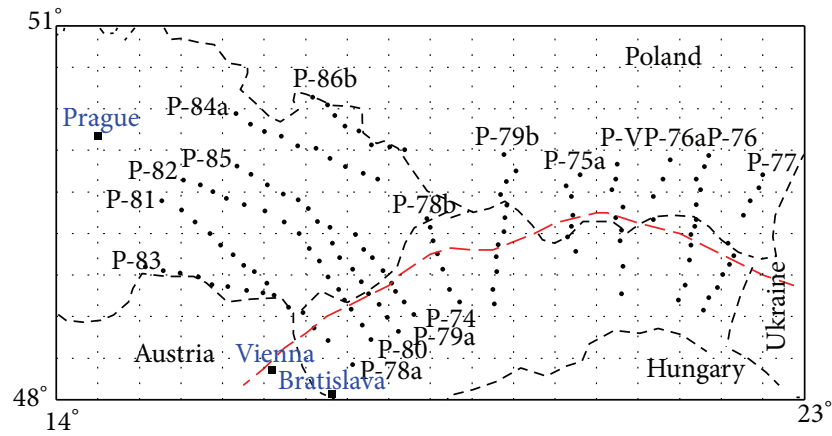

FIGURE 3: The study area shown in WGS84 geographical coordinates system along with GDS stations (dots), country border outline (thin dashed line), the West Carpathian anomaly (red dashed line), and discretization grids (thin dotted line) of 9 by 18 blocks with 35 by $35 \mathrm{~km}$ width for the thin-sheet modeling.

and 96 minutes (not shown), exhibit similar characteristics to those presented in Figure 4.

For the inversion, instead of interpolating the GDS data into more regular grid, for each block, we selected the datum located closest to the center of the block. In addition, we chose the most representative and coherent datum by evaluating the difference in a least-squared sense of the datum with its neighbors at the same block (if they exist). In this way we avoid introducing spurious effects that may be caused by interpolation process. However, there were blocks with no data at all that will be less constrained in the inversion process (see Figure 5).
From previous studies [11] and also from the results of Kováčiková et al. [15] we established a stratified (1D) medium for the host of a heterogeneous layer representing conductivity variation of the study area. The thin-sheet is $10 \mathrm{~km}$ thick at $20 \mathrm{~km}$ depth with a normal resistivity of $500 \mathrm{Ohm} \cdot \mathrm{m}$ or equivalent to a conductance of $20 \mathrm{Siemens} / \mathrm{m}$. The thin-sheet was discretized into 9 by 18 blocks of 35 by $35 \mathrm{~km}$ width (see Figure 3). The choice was dictated by the validity of the thinsheet approximation; that is, the thickness of blocks should be less than their horizontal extension and also by the current available computation resources. Based on our experience with synthetic data [9], we used "a priori" conductance values from 10 up to 2000 Siemens/m discretized into quasilogarithmic intervals to represent resistive to conductive anomalies in the region.

\section{Results and Discussion}

Starting from normal conductance (i.e., 20 Siemens/m) the inversion was performed up to 20 iterations. The posterior model was obtained by averaging the conductances of each block from the last 15 iterations. We present in Figure 5 the comparison between the calculated and observed data only for the real part of the induction arrows, since their physical correlation to the conductive anomaly is more obvious. In general, the fit is relatively good both for arrows' direction and magnitude. In Figure 5, when both observed and calculated induction arrows coincide, although their magnitude might be different (in this case smaller), then it appears that only data are plotted. 


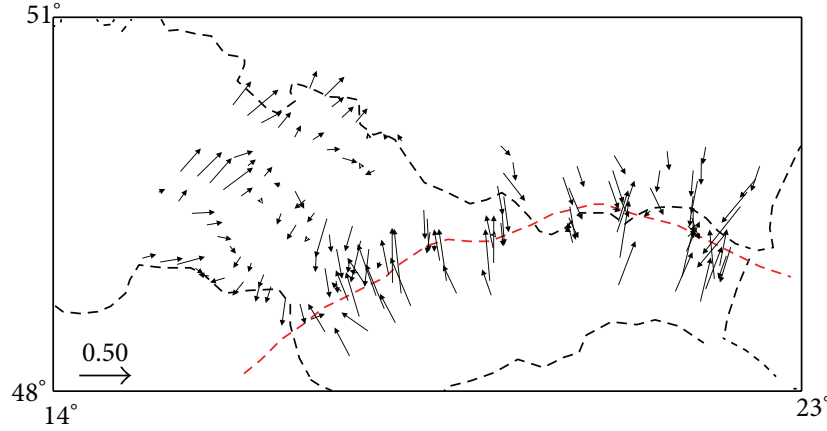

(a)

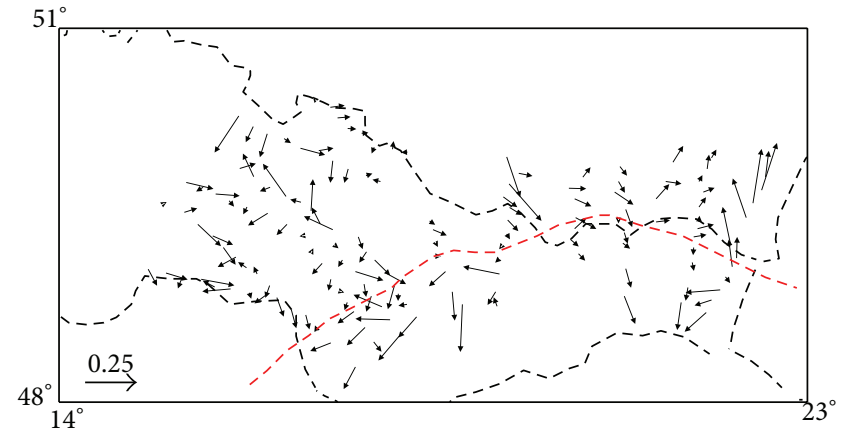

(b)

FIGURE 4: The GDS data represented as real part (a) and imaginary part (b) of the magnetic inductions vectors. The real part was inverted in accordance with Parkinson's convention such that the induction arrows point towards more conductive zones. The reference magnitude of the induction arrows is given in the bottom-left corner of each map.

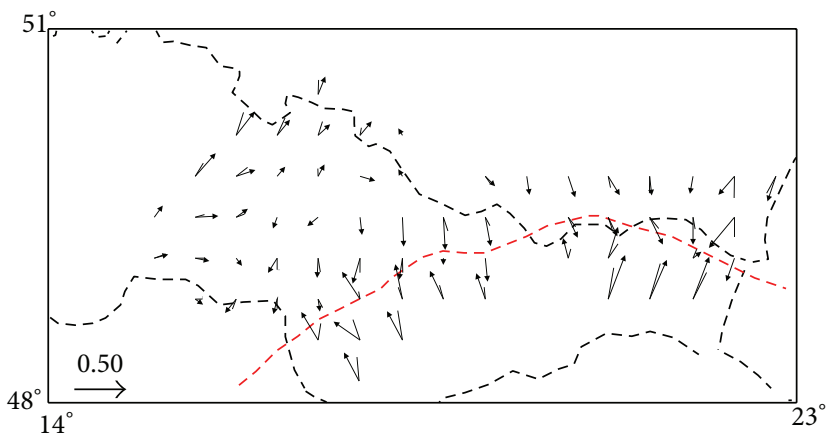

FIGURE 5: The real part of GDS data (arrows) and the calculated data (arrows without arrowhead) showing the fitness of the model response to the observed data. In blocks with only data plotted, both observed and calculated induction arrows coincide although their magnitude might be different.

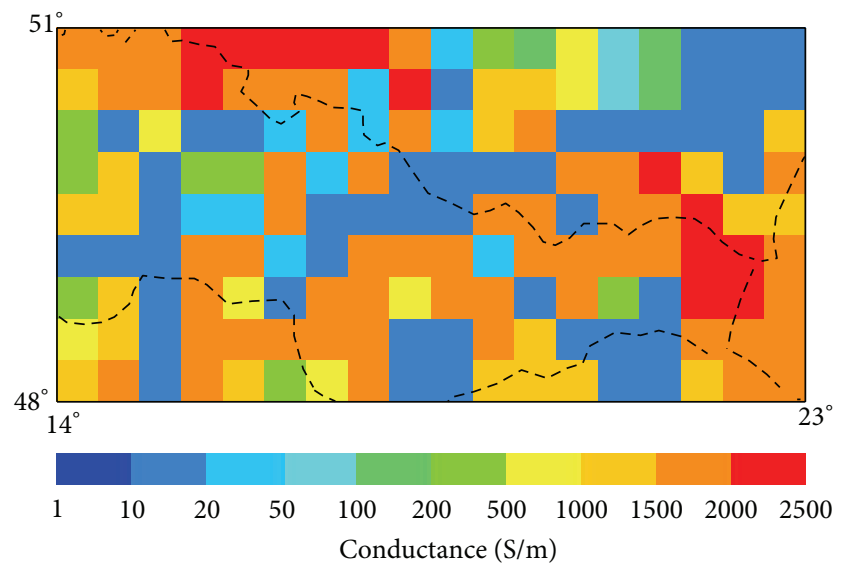

(a)

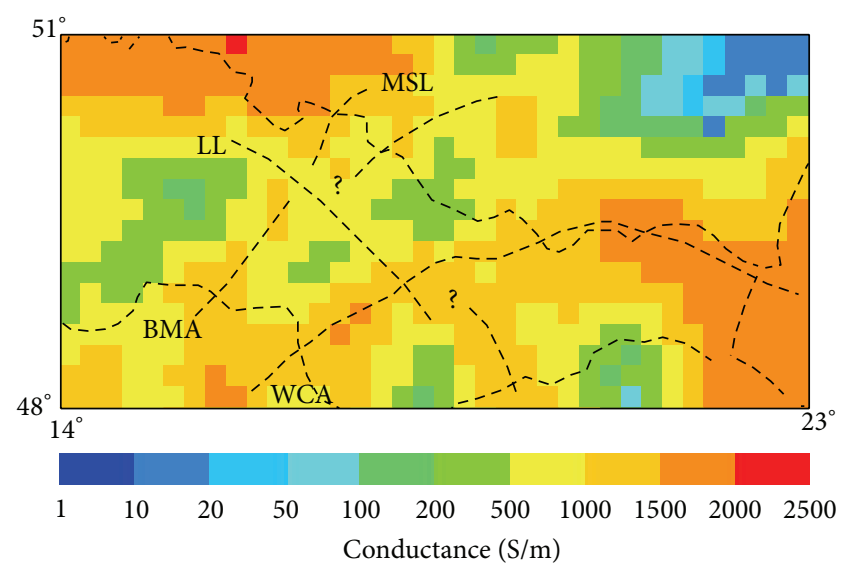

(b)

FIGURE 6: Conductance map from inversion of GDS data with initial block size (a) and after rediscretization of blocks (b). Interpreted anomalies include West Carpathian anomaly (WCA), Bohemian Massif anomaly (BMA), Moravian-Silesian lineament (MSL), and Labe lineament (LL).

The inverse model is presented in Figure 6. Despite the relatively rapid convergence of the inverse models, significant spatial variation of conductance is observed on the average model (see Figure 6(a)). The spatial discretization of the thinsheet did not allow for a more regular conductance variation from block to block. In this case, additional constraint equivalent to the smoothness constraint as in $1 \mathrm{D}$ inversion [3-6] would be difficult to apply. It requires to discretize the thin-sheet into a large number of blocks resulting in blocks mostly with no data. On the other hand, the thin-sheet 
approximation imposes minimum block size compared to the thickness of the thin-sheet and the depth of penetration of the EM waves, such that the approximation is still valid.

In order to propose more meaningful interpretation, we rediscretized the thin-sheet conductance map obtained from the inversion into blocks with a half of their initial size, that is, approximately 17.5 by $17.5 \mathrm{~km}$. The new interpolated conductance is obtained by employing a simple 5 by 5 block 2D moving average filter. The resulting model (see Figure 6(b)) has a more significant meaning as it offers the possibility of correlation with the geological features in the area. In general, our result shows that the middle and lower crust between 20 and $30 \mathrm{~km}$ depth are mostly conductive. We can also observe relatively narrow conductive zones (between 1000 and 1500 Siemens/m) which separates more resistant blocks (from 100 to 1000 Siemens/m).

The result confirms the existence of the well-known conductivity anomalies, that is, the Bohemian Massif anomaly (BMA) and the West Carpathian anomaly (WCA). More detailed examination of the result shows that there is a lateral shift between the surface curved features of the West Carpathians and the conductive anomaly from the model. This anomaly is interpreted rather as the effect of the root of the dipping contact between geological entities forming the West Carpathians at depth (more than $10 \mathrm{~km}$ ). This may also reflect the fact that we determine the integrated conductivity of the thin plate, which may include the contribution of anomalies located at different depths between 20 and $30 \mathrm{~km}$.

Our model also highlights conductive zones other than previously known anomalies. By considering also the qualitative interpretation of the induction vector maps, other less obvious anomalies found coincide with the tectonic features present in this area such as the Moravian-Silesian lineament (MSL) and the Labe lineament (LL) [18]. Furthermore, we can observe a significant conductivity anomaly at an area to the south of the West Carpathians. Although the inverse model in that area is not sufficiently constrained by the data, the existence of such anomaly would correspond to significant conductivity heterogeneities in the northern part of the Pannonian Basin [19]. Nevertheless, we could not interpret rigorously the significance of the conductive zones at northern and eastern parts of the study area and the resistive one to the north-east. These variations of conductance are lacking of geological evidences and are not well constrained by the observed data.

The resistivity corresponding to the conductance of the anomaly of a thin plate with thickness of $10 \mathrm{~km}$ is less than $10 \mathrm{Ohm} \cdot \mathrm{m}$. Other estimates indicate the resistivity lower than $5 \mathrm{Ohm} \cdot \mathrm{m}$ resistivity. Several hypotheses have been proposed to explain the presence of such a conducting medium in a very resistive host (between 500 and $1000 \mathrm{Ohm} \cdot \mathrm{m}$ ) more than $20 \mathrm{~km}$ deep. One that is most plausible is to assume that the conductive body is sedimentary rocks saturated with saline water. The presence of saline water at high temperature in porous rocks greatly increases the conductivity of the latter. This hypothesis favoured in Jankowski et al. [20] is supported in particular by recent studies on the origin of the conductivity anomaly generally observed in the lower crust [21].

\section{Conclusions}

The thin-sheet modeling employing MCMC inversion algorithm has been applied to GDS data from the area covering the Bohemian Massif and the West Carpathians with satisfactory result. The conductance map obtained from such inversion modeling correlates well with the regional geology of the study area. The result allows more quantitative analysis of the conductivity anomalies present in the area rather than only qualitative interpretation based on the pattern of the induction vectors or contoured map of the magnetic transfer function values.

The MCMC algorithm applied in this study is relatively generic and would be applicable to most geophysical inverse problems with appropriate parameterization. However, the MCMC algorithm remains a computer intensive method since it has to resolve the forward modeling a substantial number of times to explore the model space. Due to limited number of iterations that can be performed with our current computation resources, the posterior quantities leading to the model uncertainty is still underexploited. With the advances in computation processing power, in the near future, it would be possible to perform more thorough analysis of the inverse model uncertainty from the posterior probability function. It would be also possible to employ a full 3D EM modeling to avoid limitation of the thin-sheet modeling in representing complex subsurface heterogeneities.

\section{Acknowledgments}

The authors greatly acknowledge Václav Červ and his colleagues from the Institute of Geophysics, Prague, Czech Republic, for providing the GDS data for this study.

\section{References}

[1] G. Vasseur and P. Weidelt, "Bimodal electromagnetic induction in non-uniform thinsheets with an application to the northern Pyrenean inductionanomaly," Geophysical Journal of Royal Astronomical Society, vol. 51, no. 3, pp. 669-690, 1977.

[2] J. Sun and G. D. Egbert, "A thin-sheet model for global electromagnetic induction," Geophysical Journal International, vol. 189, no. 1, pp. 343-356, 2012.

[3] H. Grandis, M. Menvielle, and M. Roussignol, "Bayesian inversion with Markov chains-I. The magnetotelluric onedimensional case," Geophysical Journal International, vol. 138, no. 3, pp. 757-768, 1999.

[4] O. Praus, J. Pěčová, V. Červ, S. Kováčiková, J. Pek, and J. Velímský, "Electrical conductivity at mid-mantle depths estimated from the data of Sq and long period geomagnetic variations," Studia Geophysica et Geodaetica, vol. 55, no. 2, pp. 241-264, 2011.

[5] J.-J. Schott, M. Roussignol, M. Menvielle, and F. R. Nomenjahanary, "Bayesian inversion with Markov chains-II. The onedimensional DC multilayer case," Geophysical Journal International, vol. 138, no. 3, pp. 769-783, 1999.

[6] H. Grandis and P. Sumintadireja, "Quasi-2D resistivity model from inversion of CSAMT (Controlled-Source Audiofrequency Magnetotellurics) data," in Proceedings of the HAGI-IAGI Joint Convention Medan, 2013. 
[7] L. J. Wang and F. E. M. Lilley, "Inversion of magnetometer array data by thin-sheet modelling," Geophysical Journal International, vol. 137, no. 1, pp. 128-138, 1999.

[8] D. McA. Mckirdy, J. T. Weaver, and T. W. Dawson, "Induction in a thin sheet of variable conductance at the surface of a stratified earth-II. Three-dimensional theory," Geophysical JournalRoyal Astronomical Society, vol. 80, no. 1, pp. 177-194, 1985.

[9] H. Grandis, M. Menvielle, and M. Roussignol, "Thin-sheet electromagnetic inversion modeling using Monte Carlo Markov Chain (MCMC) algorithm," Earth, Planets and Space, vol. 54, no. 5, pp. 511-521, 2002.

[10] F. E. M. Lilley and B. R. Arora, "The sign convention for quadrature Parkinson arrows in geomagnetic induction studies," Reviews of Geophysics \& Space Physics, vol. 20, no. 3, pp. 513-518, 1982.

[11] H. Grandis, Imagerie electromagnetique Bayesienne par la simulation d'une chaîne de Markov [Ph.D. thesis], Sciences de la Terre et des Planètes, Université Paris 7, Paris, France, 1994.

[12] D. W. Heerman, Computer Simulation Methods in Theoretical Physics, Springer, Berlin, Germany, 1990.

[13] C. Robert, Méthodes de Monte Carlo par Chaînes de Markov, Economica, Paris, France, 1996.

[14] A. Dudek, "The principal features of the geological structure of Czecho-Slovakia with regard to adjacent regions," in Geophysical Syntheses in Czecho-Slovakia, A. Zátopek, Ed., Veda, Bratislava, Slovakia, 1981.

[15] S. Kováčiková, V. Červ, and O. Praus, "Modelling of the conductance distribution at the eastern margin of the European Hercynides," Studia Geophysica et Geodaetica, vol. 49, no. 3, pp. 403-421, 2005.

[16] K. Pěč, J. Pěčová, O. Praus, and M. Hvoždara, "Single- and interstation transfer functions for non-synchronous geomagnetic arrays-I. Theory of methods used in data analysis," Studia Geophysica et Geodaetica, vol. 32, no. 3, pp. 300-312, 1988.

[17] K. Pěč, J. Pěčová, O. Praus, and M. Hvoždara, "Single- and interstation transfer functions for non-synchronous geomagnetic arrays-II. the results of the field data analysis," Studia Geophysica et Geodaetica, vol. 32, no. 4, pp. 368-391, 1988.

[18] V. Petr, J. Pěčová, O. Praus, and K. Pěč, "Anomalous induction zone near the eastern margin of the Bohemian Massif," Physics of the Earth and Planetary Interiors, vol. 45, no. 2, pp. 161-169, 1987.

[19] A. Ádám and A. Koppán, "Long period electromagnetic induction vectors in a sedimentary back-arc basin (CarpathoPannonian Basin)," Acta Geodaetica et Geophysica Hungarica, vol. 39, no. 4, pp. 363-379, 2004.

[20] J. Jankowski, O. Praus, and W. Jóźwiak, "Carpathian anomaly of electrical conductivity: history of its discovery and interpretation," Publications of the Institute of Geophysics-Polish Academy of Sciences, vol. 95, no. 386, pp. 17-27, 2005.

[21] R. D. Hyndman, L. L. Vanyan, G. Marquis, and L. K. Law, “The origin of electrically conductive lower continental crust: saline water or graphite?" Physics of the Earth and Planetary Interiors, vol. 81, no. 1-4, pp. 325-345, 1993. 

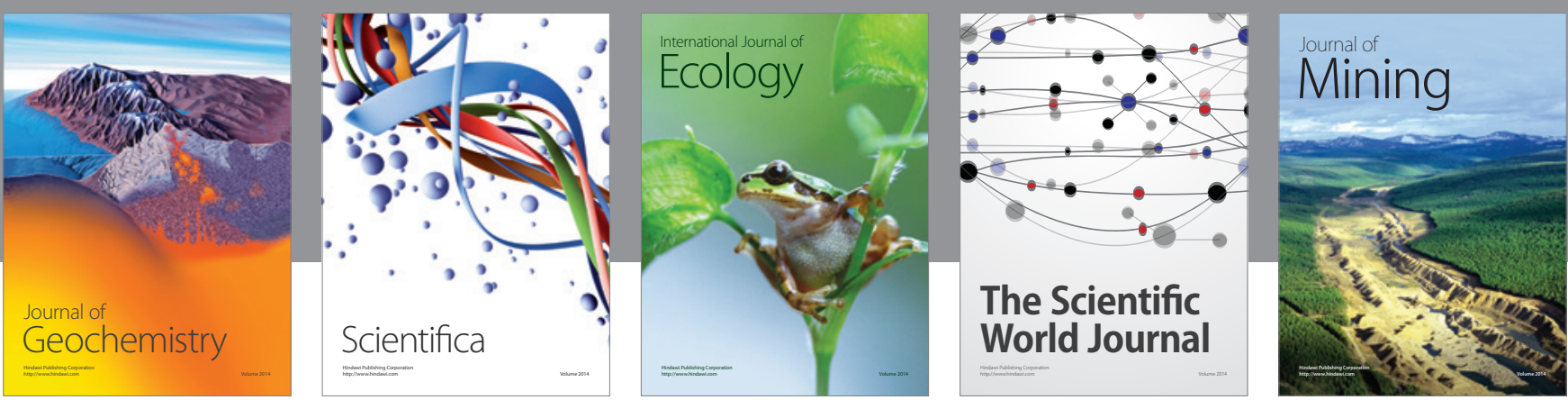

The Scientific World Journal
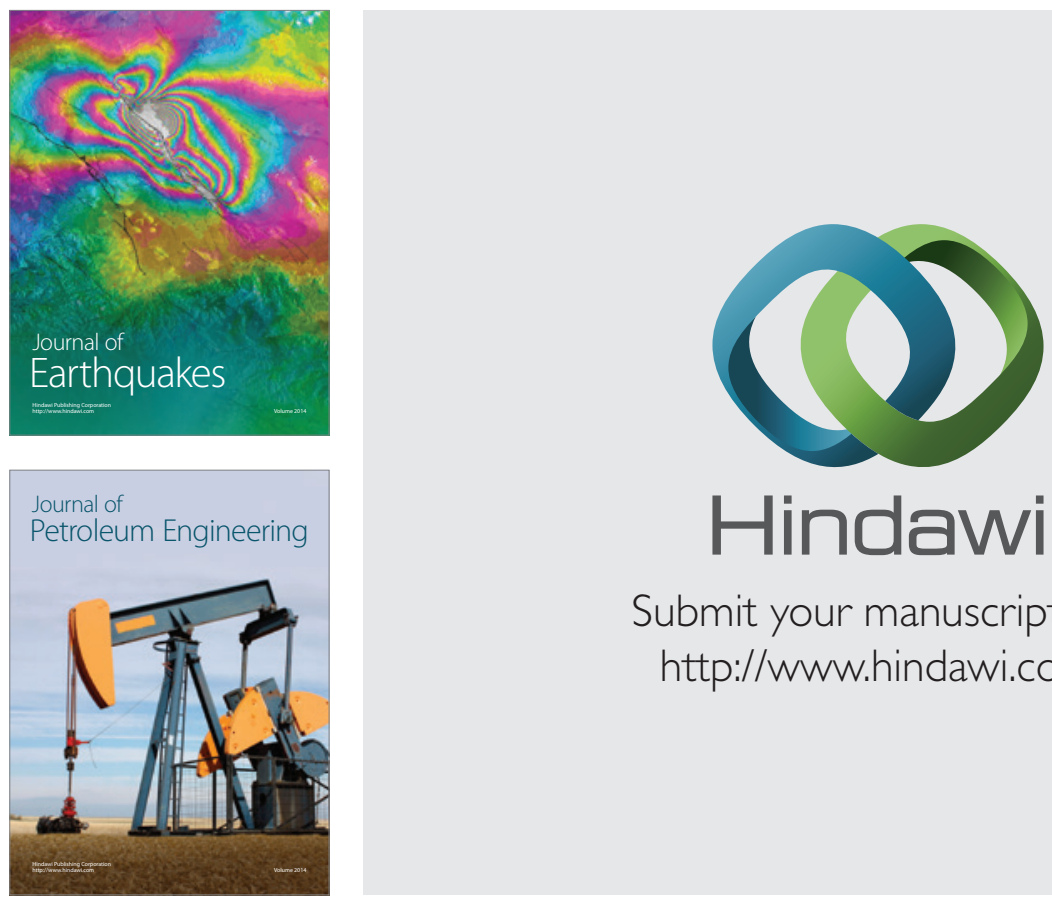

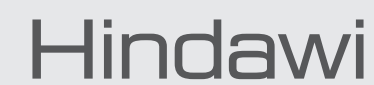

Submit your manuscripts at

http://www.hindawi.com
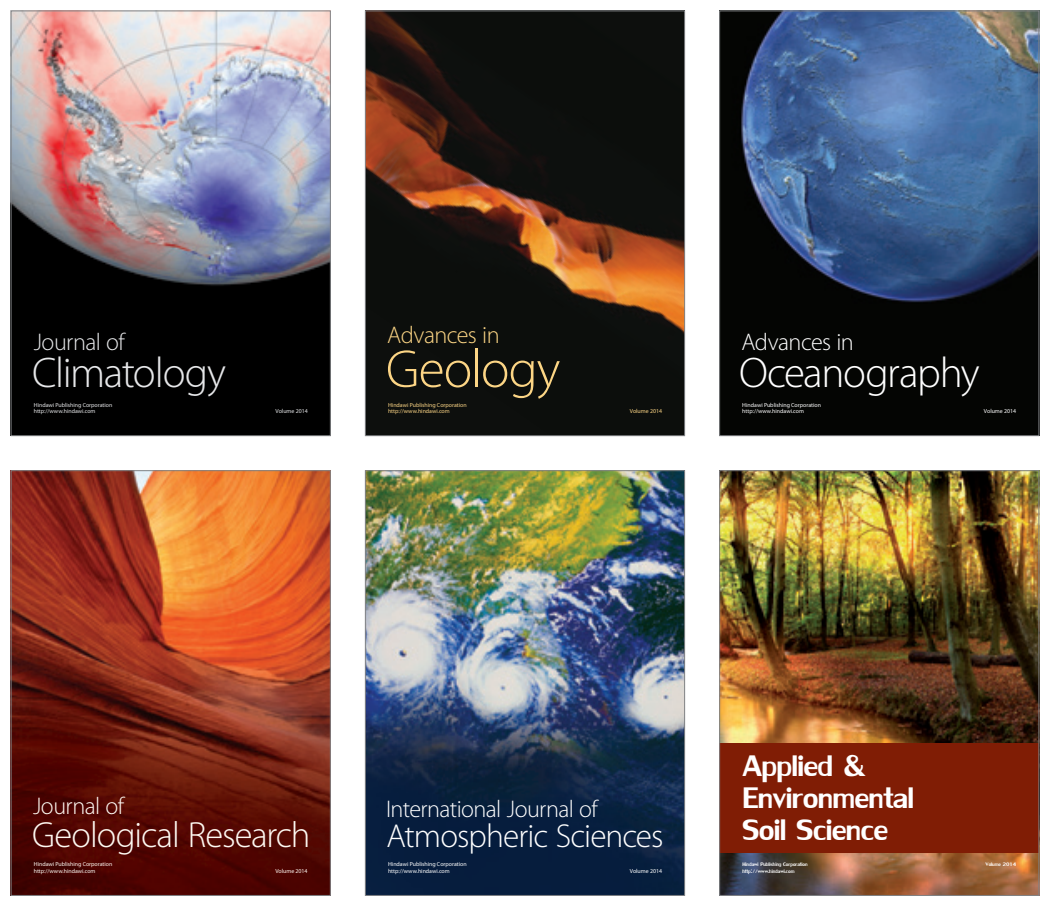
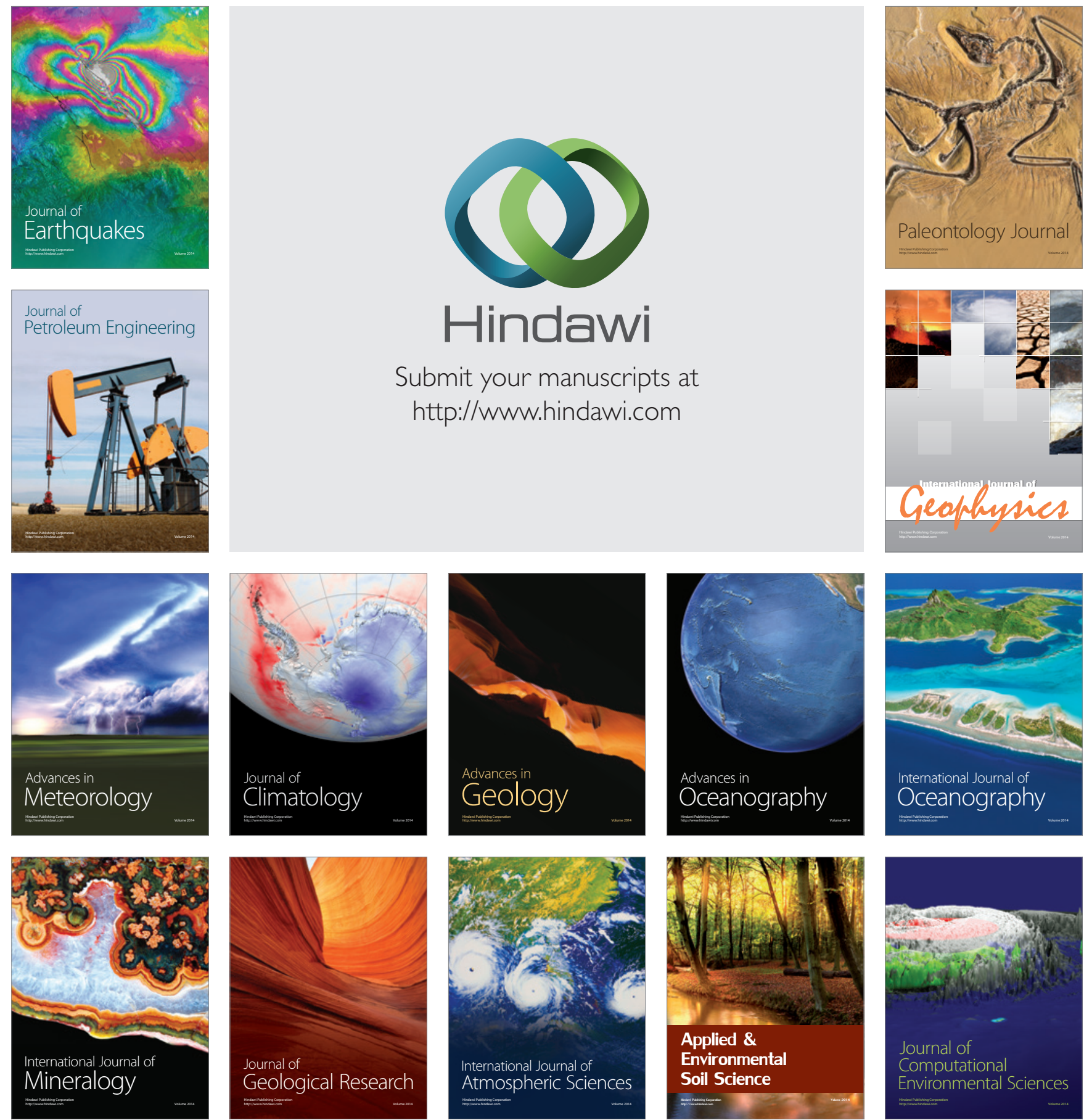